 \\ MOUNTaiNS \\ Mathematical Publications
}

\section{CAUCHY FUNCTIONAL EQUATION AND GENERALIZED CONTINUITY}

\author{
PAvel Kostyrko
}

\begin{abstract}
There are many kinds of the generalization of continuity. T. Salát raised the question: Can everywhere discontinuous solution of Cauchy functional equation

$$
f(x+y)=f(x)+f(y)
$$
\end{abstract}

be continuous in some generalized sense? The paper deals with this question.

\section{Introduction}

The paper deals with the Cauchy functional equation

$$
f(x+y)=f(x)+f(y)
$$

for $f: \mathbb{R} \rightarrow \mathbb{R} \quad(\mathbb{R}$ - the real line). This equation is well-known, see [9], and its solutions are called additive functions. It is known that every solution of (1) is either of the form $f(x)=x f(1)$ for each $x \in \mathbb{R}$ or $f$ is discontinuous everywhere on $\mathbb{R}$.

There are many kinds of the generalization of the notion of continuity. T. Šalát raised the following question: Can everywhere discontinuous solution of (1) be continuous in some generalized sense? The following text is devoted to this question.

Further, we will use some basic properties of additive functions (see [9]). The Hamel base for $\mathbb{R}$ is a subset $H$ of $\mathbb{R}$ with the property that each non-zero element $x$ of $\mathbb{R}$ is representable in a unique way as a finite linear combination of distinct members of $H$, with non-zero rational coefficients, i.e.,

$$
x=r_{1} h_{1}+\cdots+r_{n} h_{n},
$$

2000 Mathematics Subject Classification: 26A15, 26A99.

Keywords: Cauchy functional equation, generalized continuity.

Supported by the Grand VEGA 1/3018/06. 
PAVEL KOSTYRKO

where $h_{1}, \ldots, h_{n} \in H$ and $r_{1}, \ldots, r_{n} \in \mathbb{Q} \quad(\mathbb{Q}-$ rationals $)$. If $g: H \rightarrow \mathbb{R}$ is an arbitrary function, then its extension on $\mathbb{R}$ given by

$$
f(x)=r_{1} g\left(h_{1}\right)+\cdots+r_{n} g\left(h_{n}\right)
$$

( $x$ is expressed by (2) ) is an additive function and every additive function is of this form.

Lemma K. Let $f: \mathbb{R} \rightarrow \mathbb{R}$ be an additive function bounded on some set of positive Lebesgue measure. Then $f(x)=x f(1)$ for each $x \in \mathbb{R}$.

Proof. See [7].

Lemma G. Let $f: \mathbb{R} \rightarrow \mathbb{R}$ be a discontinuous additive function. Then its graph is a dense subset of $\mathbb{R} \times \mathbb{R}$.

Proof. See [5], [10, p. 137].

Further, let CFE stand for the set of all additive functions $f: \mathbb{R} \rightarrow \mathbb{R}$.

\section{Definitions}

The following definition gives some of known generalizations of continuity. We will deal with them.

Definition 1. Let $f: \mathbb{R} \rightarrow \mathbb{R}$.

(a) A function $f$ is said to be symmetrically continuous if for each $x \in \mathbb{R}$ $\lim _{h \rightarrow 0}(f(x+h)-f(x-h))=0$ (see [20]).

(b) A function $f$ is said to have the closed graph if $\{(x, f(x)): x \in \mathbb{R}\}$ is a closed subset of $\mathbb{R} \times \mathbb{R}$ (see [8]).

(c) A function $f$ is said to be quasicontinuous if for each $x \in \mathbb{R}$ it holds: If $V$ is a neighborhood of $f(x)$, then $x \in \mathrm{Cl} \operatorname{Int} f^{-1}(V)$ (see [11]).

(d) A function $f$ is said to be cliquish if for each $x \in \mathbb{R}$ holds: If $\varepsilon>0$ and $U$ is a neighborhood of $x$, then there is an open set $G \subset U$ such that $\mid f(r)-$ $f(s) \mid<\varepsilon$ holds for any $r, s \in G$ (see [2]).

(e) A function $f$ is said to be simply continuous if for any open set $G, f^{-1}(G)$ is the union of an open set and a nowhere dense set (see [1]).

(f) A function $f$ is said to be somewhat continuous if for each open set $V \subset \mathbb{R}$, $f^{-1}(V) \neq \emptyset$ implies Int $f^{-1}(V) \neq \emptyset$ (see [17]). 


\section{CAUCHY FUNCTIONAL EQUATION AND GENERALIZED CONTINUITY}

(g) A function $f$ is said to have the Denjoy property if for each $a, b \in \mathbb{R}, a<b$, the set $M_{a, b}=\{x \in \mathbb{R}: a<f(x)<b\}$ is either void or there exists $A \subset M_{a, b}$ such that $\lambda(A)>0(\lambda$ - Lebesgue measure) (see [19]).

(h) A function $f$ is said to be almost continuous in the sense of Signal and Signal if for each $x \in \mathbb{R}$ and each neighborhood $V$ of $f(x)$ there is an open set $U, x \in U$, such that $f(U) \subset \operatorname{Int} \mathrm{Cl}(V)$ (see [16]).

(i) A function $f$ is said to be weakly continuous at $x \in \mathbb{R}$ if for each neighborhood $V$ of $f(x)$ there is an open set $U, x \in U$, such that $f(U) \subset \mathrm{Cl}(V)$ ([13]).

(j) A function $f$ is said to be symmetric if $\lim _{h \rightarrow 0}(f(x+h)+f(x-h)-2 f(x))=0$ holds for each $x \in R([20])$.

(k) A function $f$ is said to be almost continuous in the sense of Stallings, if each neighborhood of its graph contains a graph of a continuous function $([18)$.

(l) A function $f$ is said to be locally almost continuous if $x \in \operatorname{Int} \mathrm{Cl}^{-1}(V)$ holds for each $x \in \mathbb{R}$ and any neighborhood $V$ of $f(x)([12])$.

(m) A function $f$ is said to be locally almost quasicontinuous if $x \in$ $\mathrm{Cl}$ Int $\mathrm{Cl} f^{-1}(V)$ holds for each $x \in \mathbb{R}$ and any neighborhood $V$ of $f(x)$ $([12])$.

(n) A function $f$ is said to be graph continuous if the closure of its graph contains the graph of a continuous function ([4]).

(o) A function $f$ is said to be Darboux continuous if for each $x_{1}, x_{2} \in \mathbb{R}$, $x_{1}<x_{2}, f\left(x_{1}\right) \neq f\left(x_{2}\right)$ and $y \in \mathbb{R}$ between $f\left(x_{1}\right)$ and $f\left(x_{2}\right)$ there exists $x, x_{1}<x<x_{2}$, such that $y=f(x)$.

Remark 1. All the generalized continuities mentioned in Definition 1 are known from the literature. The only exception is the definition (g) of the Denjoy property, which spreads the known definition on functions Lebesgue nonmeasurable.

\section{DeFinition 2.}

(1) Let $(z)$ be some of the generalized continuities from Definition 1 The symbol $Z$ stands for the family of all functions $f: \mathbb{R} \rightarrow \mathbb{R}$ continuous in the sense $(z)$.

(2) We will say that the generalized continuity $(z)$ is of type A if each additive function which is $(z)$ generalized continuous is of the form $f(x)=c x$, $c \in \mathbb{R}$.

(3) A generalized continuity $(z)$ is said to be of type B if it is not of type A. 
PAVEL KOSTYRKO

\section{Results}

Theorem 1. Generalized continuities (a), (b), (c), (d), (e), (f), (g), (h) and (i) are of type $A$.

Theorem 2. Generalized continuities (j), (k), (l), (m), (n) and (o) are of type B.

Proof of Theorem 1. Let $(z)$ be some of the generalized continuities mentioned in Theorem 1, To prove Theorem 1, it is sufficient to show

$$
C F E \cap Z=W,
$$

where $W$ is the set of all the functions $f: \mathbb{R} \rightarrow \mathbb{R}$ of the form $f(x)=c x, c \in \mathbb{R}$.

(a) Each symmetrically continuous function is continuous almost everywhere (see [20], 14]), the assumptions of Lemma K are fulfilled and (3) follows.

(b) Each function with the closed graph belongs to Baire class 1, it has continuity points and Lemma K implies (3).

(c) Let $x \in \mathbb{R}$ and $V$ be a bounded neighborhood of $f(x)$. From the definition of quasicontinuity of $f$ at $x$, it follows that $f$ is bounded on Int $f^{-1}(V) \neq \emptyset$, i.e., (3) is a consequence of Lemma K.

(d) The argumentation over cliquish functions is analogous to the case (c).

(e) The argumentation over simply continuous functions is analogous to the case (c).

(f) We choose $V=(-1,1)$. Obviously, $f^{-1}(V) \neq \emptyset$. Since $f$ is somewhat continuous, Int $f^{-1}(V) \neq \emptyset$. The equality (3) is a consequence of Lemma K.

(g) Put $a=-1$ and $b=1$. Then $M_{a, b} \neq \emptyset$ and since $f$ has the Denjoy property, there is $T \subset M_{a, b}$ with $\lambda(T)>0$ and Lemma $\mathrm{K}$ implies (3)).

(h) The idea of the proof is the same as in the case (c).

(i) The idea of the proof is the same as in the case (c).

Proof of Theorem 2, We show that for every generalized continuity $(z)$, mentioned in Theorem 2, with the exception of Darboux continuity (o), $C F E \subset Z$ holds.

(j) If $f \in C F E$, then for each $x$ and $h$ we have $f(x+h)+f(x-h)-2 f(x)=0$ and $f \in J$.

(k) If $f \in C F E$ is discontinuous, then the graph of $f$ is dense in $\mathbb{R} \times \mathbb{R}$ (Lemma $G$ ) and each its neighborhood is of the form $\mathbb{R} \times \mathbb{R}$. It contains a graph of any continuous function, hence $f \in K$. 


\section{CAUCHY FUNCTIONAL EQUATION AND GENERALIZED CONTINUITY}

(l) If $f \in C F E$ is discontinuous, $(a, b) \subset \mathbb{R}$ is an arbitrary interval and $p \in \mathbb{R}$. If $V$ is an open neighborhood of $f(p)$, then from the density of the graph of $f$ in $\mathbb{R} \times \mathbb{R}$ it follows, that there is $x \in(a, b)$ such that $(x, f(x)) \in(a, b) \times V$. Hence $x \in f^{-1}(V)$. Consequently, $f^{-1}(V)$ is dense in $\mathbb{R}, \mathrm{Cl}^{-1}(V)=\mathbb{R}$, Int $\mathrm{Cl}^{-1}(V)=\mathbb{R}$ and $p \in \operatorname{Int} \mathrm{Cl} f^{-1}(V)=\mathbb{R}$, i.e., $f \in L$.

(m) The inclusion $C F E \subset M$ follows from the part (1). It is sufficient to take $\mathbb{R}=\operatorname{Int~} \mathrm{Cl}^{-1}(V) \subset \mathrm{Cl}$ Int $\mathrm{Cl} f^{-1}(V)$ into account.

(n) If $f \in C F E$ is discontinuous, then the closure of its graph is $\mathbb{R} \times \mathbb{R}$ and it contains the graph of a continuous function.

(o) We show that there exist a discontinuous function $f \in C F E \cap O$. Let $\left(H_{n}\right)_{n=1}^{\infty}$ be such a decomposition of the Hamel basis $H$ that each $H_{n}$ has cardinality $c$, i.e., $H=\bigcup_{n=1}^{\infty} H_{n}, H_{n} \cap H_{m}=\emptyset$ for $n \neq m$ and card $H_{n}=c$ for each $n=1,2, \ldots$ Let $\left(I_{n}\right)_{n=1}^{\infty}$ be a sequence of all intervals with rational endpoints. For a fixed $n$, let $\varphi_{n}: H_{n} \rightarrow \mathbb{R}$ be a surjective map. For each $x \in H_{n}$ there is $r_{x} \in \mathbb{Q}, r_{x} \neq 0$, such that $r_{x} x \in I_{n}$. For $x \in H_{n}$ define $f_{n}(x)=\varphi_{n}(x) / r_{x}$. Let $f: H \rightarrow \mathbb{R}$ be defined by $f(x)=f_{n}(x)$ for $x \in H_{n}$ and let $F$ be an additive extension of $f$ on $\mathbb{R}$. Let $a<b$ and $y \in \mathbb{R}$. Choose $n$ such that $I_{n} \subset(a, b)$. There is $x \in H_{n}$ such that $y=\varphi_{n}(x)$ and $r_{x} \in \mathbb{Q}$ with $r_{x} x \in I_{n}$. Then, $F\left(r_{x} x\right)=r_{x} F(x)=r_{x} f(x)=r_{x} f_{n}(x)=\varphi_{n}(x)=y$. Hence, $F((a, b))=\mathbb{R}$ and $F \in C F E \cap O$.

Remark 2. There are functions $g: \mathbb{R} \rightarrow \mathbb{R}$ such that $g \in C F E \backslash O$. If suffices to define $g$ on the Hamel basis of rational numbers.

Remark 3. There is a natural question: Is the inclusion $C F E \subset Z$ in the proof of Theorem 2 proper? The answer is affirmative. If follows from the fact that the generalized continuities from Theorem 2 are invariant with respect to the vertical translation, i.e., if $f \in Z$ and $\alpha \in \mathbb{R}$, then $f+\alpha \in Z$.

Let $f: \mathbb{R} \rightarrow \mathbb{R}$ be a discontinuous additive function and $\alpha \neq 0$. Put $f_{\alpha}(x)=$ $f(x)+\alpha$. Then $f_{\alpha}$ is not additive $\left(f_{\alpha}(0)=\alpha\right)$ and $f_{\alpha} \in Z$.

\section{REFERENCES}

[1] BORSÍK, J.-DOBOŠ, J.: On simple continuity points, Real Anal. Exchange 16 (1991), $552-558$.

[2] DOBOŠ, J.: Simple continuity and cliquishnees, Čas. Pěst. Mat. 112 (1987), 355-358.

[3] GENTRY, K. R.-HOYLE, H. B.: Somewhat continuous functions, Czechoslovak. Math. J. 21 (1971), 5-12.

[4] GRANDE, Z.: Sur les fonctions A-continues, Demonstratio Math. 11 (1978), 519-526. 


\section{PAVEL KOSTYRKO}

[5] JONES, F. B.: Connected and disconnected plane sets and the functional equation $f(x+y)=f(x)+f(y)$, Bull. Amer. Math. Soc. 48 (1942), 115-120.

[6] KELlUM, K. R.: Sums and limits of almost continuous functions, Colloq. Math. 31 (1974), 125-128.

[7] KESTELMAN, H.: On the equation $f(x+y)=f(x)+f(y)$, Fund. Math. 34 (1947), 144-147.

[8] KOSTYRKO, P.- ¿̌́ALÁT, T.: On functions with closed graphs, Čas. Pěst. Mat. 89 (1984), 426-432. (In Russian)

[9] KUCZMA, K.: An Introduction to the Theory of Functional Equations and Inequalities, PWN, Warszawa, 1965.

[10] LUKEŠ, J. A KOL.: Problémy z Matematické Analýzy, SPN, Praha, 1972. (In Czech)

[11] MARCUS, S.: Sur le fonctions quasicontinues au sens de S. Kempisty, Colloq. Math. 8 (1961), 47-53.

[12] NEUBRUNNOVÁ, A.- ŠALÁT, T.: On almost quasicontinuity, Math. Bohem. 117 (1992), 197-205.

[13] NOIRI, T.: On weakly continuous mappings, Proc. Amer. Math. Soc. 46 (1974), 120-124.

[14] PREISS, D.: A note on symmetrically continous mappings, Čas. Pěst. Mat. 96 (1971), 262-264.

[15] ROSEN, H.: On characterizing extentable conectivity functions by associated sets, Real Anal. Exchange 22 (1996-97), 279-283.

[16] SINGAL, M. K.-SINGAL, A. R.: Almost continuous mappings, Yokohama Math. J. 16 (1968), 63-73.

[17] SMÍTAL, J.- ¿̌SALÁT, T.: Remarks on two generalizations of the notion of continuity, Acta Fac. Rer. Nat. Univ. Com. 36 (1980), 115-116.

[18] STALLINGS, J.: Fixed point theorem for connectivity maps, Fund. Math. 47 (1959), 249-263.

[19] ŠALÁT, T.: Some generalizations of the notion of continuity and Denjoy property of functions, Čas. Pěst. Mat. 99 (1974), 380-385.

[20] THOMSON, B. S.: Symmetric Properties of Real Functions, Marcel Dekker, New York, 1994.

Received November 10, 2008

Comenius University

Faculty of Mathematics,

Physics and Informtics

Department of Mathematics

SK-842-48 Bratislava 4

SLOVAKIA

E-mail: kostyrko@fmph.uniba.sk 